\title{
PEPS as unique ground states of local Hamiltonians
}

\author{
D. Pérez-García \\ Departamento de Analisis Matematico. Universidad Complutense de Madrid, 28040 Madrid, Spain. \\ F. Verstraete \\ Fakultät für Physik, Universität Wien, Boltzmanngasse 5, A-1090 Wien, Austria. \\ J.I. Cirac and M.M. Wolf \\ Max Planck Institut für Quantenoptik, Hans-Kopfermann-Str. 1, Garching, D-85748, Germany.
}

\begin{abstract}
In this paper we consider projected entangled pair states (PEPS) on arbitrary lattices. We construct local parent Hamiltonians for each PEPS and isolate a condition under which the state is the unique ground state of the Hamiltonian. This condition, verified by generic PEPS and examples like the AKLT model, is an injective relation between the boundary and the bulk of any local region. While it implies the existence of an energy gap in the $1 \mathrm{D}$ case we will show that in certain cases (e.g., on a 2D hexagonal lattice) the parent Hamiltonian can be gapless with a critical ground state. To show this we invoke a mapping between classical and quantum models and prove that in these cases the injectivity relation between boundary and bulk solely depends on the lattice geometry.
\end{abstract}

\section{INTRODUCTION}

Exactly solvable models for quantum mechanical spin systems are important testbeds and sources of insight for the theory of condensed matter systems and their statistics. Unfortunately, such models are rare even in 1D and, disregarding free particles, essentially non-existent in higher spatial dimensions. If the focus lies in low temperature physics then quasi-exactly solvable models for which at least the ground state is known exactly become interesting. A powerful tool for constructing such models in $1 \mathrm{D}$ is the matrix product state (MPS) formalism [1, 2, 3] which has his roots in the AKLT model [4] and has experienced a remarkable development in the last years concerning both analytical results [5] and numerical methods [6]. In two and higher spatial dimensions a generalization of the MPS idea is known as projected entangled pair state (PEPS) formalism [7, 8, 9], earlier attempts of which can already be found in the seminal work of AKLT [4] as well as in studies of dimer models [10] and antiferromagnetic vertex spin models [11]. The idea of the PEPS representation of quantum states has its roots in quantum information theory [12] but it found most of its applications in quantum many-body physics [7, 8, 9, 13, 14].

The present work will continue on this line and investigate quasi-exactly solvable models based on PEPS. Our main interest lies hereby in the uniqueness of the ground state. We prove that

- every PEPS satisfying a condition we call 'injectivity' is the unique ground state of a local Hamiltonian.

- For quantum states which are coherent versions of classical Gibbs states we show that injectivity depends solely on the lattice geometry.

- By invoking the mentioned classical-to-quantum mapping [8] we construct a PEPS which is the unique critical ground state of a local Hamiltonian on a 2D hexagonal lattice. This shows that, in contrast to $1 \mathrm{D}$ MPS, injectivity does not imply the existence of an energy gap.

- We give an example on the 2D square lattice where uniqueness can be proven in the absence of injectivity.

- We provide a computable sufficient condition for the existence of an energy gap.

- In the appendix we show that every translational invariant PEPS on a 2D square lattice admits a representation with site-independent tensors.

Note that the relation between injectivity and uniqueness generalizes in a fairly direct way what is known in the 1D case 1, 2] to arbitrary lattice geometries. The main difference lies in the fact that beyond the $1 \mathrm{D}$ case the existence of a gap above the ground state energy becomes a rather independent property, the determination of which essentially remains an open problem.

\section{PRELIMINARIES}

Let us start by recalling that a PEPS is a quantum state $|\varphi\rangle$ constructed over a graph (Fig.1) such that each vertex corresponds to a physical site and the geometry of the graph typically resembles the spatial geometry of a lattice. To construct the PEPS we assign to each edge (bond) of the graph a maximally entangled state $\sum_{i=1}^{D}|i i\rangle$. In this way, each vertex gets assigned to as many $D$-dimensional systems as there are adjacent edges - we may think of these as a virtual substructure. To obtain the final state we apply a map $P: \mathcal{C}^{D} \otimes \cdots \otimes \mathcal{C}^{D} \longrightarrow \mathcal{C}^{d}$ at each vertex which maps the virtual onto the physical system. Note that $P$ and $d$ may depend on the vertex and $D$ on the edge. For 
the sake of simplicity we will, however, choose the dimensions $D$ and $d$ to be constant over the entire graph. Let us denote by $e_{v}$ the number of edges at vertex $v$ and parameterize the corresponding map $P^{(v)}$ by

$$
P^{(v)}=\sum_{i=1}^{d} \sum_{j_{1}, \ldots, j_{e_{v}}=1}^{D} A_{i, j}^{(v)}|i\rangle\left\langle j_{1}, \ldots, j_{e_{v}}\right|
$$

such that each $A_{i}^{(v)}$ is a tensor with $e_{v}$ indices $j=$ $\left(j_{1}, \ldots, j_{e_{v}}\right)$. From this we obtain a PEPS of the form

$$
|\varphi\rangle=\sum_{i_{1}, i_{2}, \ldots=1}^{d} \mathcal{C}\left[\left\{A_{i_{v}}^{(v)}\right\}_{v}\right]\left|i_{1}, i_{2}, \ldots\right\rangle,
$$

where $\mathcal{C}$ means the contraction of all tensors $A_{i}^{(v)}$ according to the edges of the graph. If, for instance, we deal with an $N \times M$ square lattice on a torus we may specify each vertex by the respective row $j$ and column $k$ such that

$$
|\varphi\rangle=\sum_{i_{(1,1)}, \ldots, i_{(N, M)}=1}^{d} \mathcal{C}\left[\left\{A_{i_{(j, k)}^{(j, k)}}^{(j, j, k)}\right]\left|i_{(1,1)} \cdots i_{(N, M)}\right\rangle\right.
$$

where each $A_{i}^{(j, k)}$ is now a 4 -index tensor which is contracted according to the lattice:

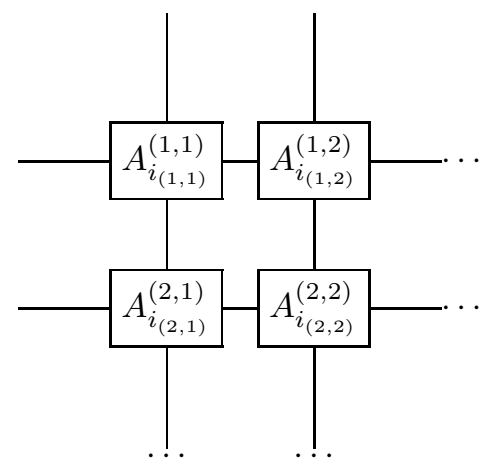

Clearly, if the tensors $A_{i}^{(j, k)}$ do not depend on the vertex $v=(j, k)$ then the corresponding PEPS $|\varphi\rangle$ is translational invariant. Conversely, if $|\varphi\rangle$ is translational invariant, then there exists always a representation (with presumably larger $D$ ) such that $A_{i}^{(j, k)}=A_{i}$ is siteindependent. A proof of this statement can be found in the appendix. We will throughout not assume such a symmetry and allow the $A_{i}$ 's to be site dependent though we typically omit to write the explicit dependence $A_{i}^{(v)}$.

\section{PARENT HAMILTONIANS}

In the following we will mainly consider lattices instead of arbitrary graphs as this gives us a meaningful notion of locality and ensures that volumes grow faster than boundary areas. The latter is, in fact, the main property
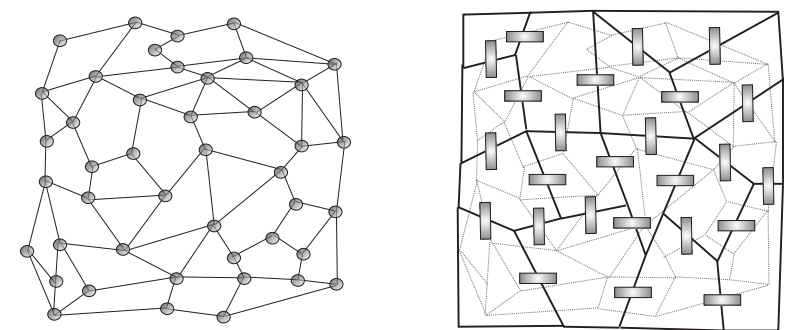

FIG. 1: Left: A PEPS can be assigned to an arbitrary graph. Each vertex corresponds to a physical site and every edge depicts the contraction of tensors assigned to the vertices. Right: A parent Hamiltonian which has the PEPS as unique ground state can be constructed by first joining vertices in injective regions (now surrounded by solid lines) and defining interactions (depicted by bars) between connected neighboring regions.

which allows us to construct parent Hamiltonians for every PEPS $|\varphi\rangle$. Consider a region $R$ on the lattice with respective reduced density operator $\rho_{R}=\operatorname{tr}_{\backslash R}|\varphi\rangle\langle\varphi|$. The support space $S_{R}$ of $\rho_{R}$ has dimension at most $D^{e_{R}}$ where $e_{R}$ is the number of edges connecting the interior of $R$ to the exterior. Denote by $|R|$ the number of lattice sites in $R$ with total Hilbert space $\mathcal{H}_{R}=\mathbb{C}^{d \otimes|R|}$. Since $S_{R}$ grows as the boundary of $R$ whereas $\mathcal{H}_{R}$ grows like its volume, we can always find a sufficiently large region such that $\rho_{R}$ will have a non-trivial kernel $\operatorname{ker}\left(\rho_{R}\right)=\mathcal{H}_{R} \backslash S_{R}$. It is thus a ground state of every Hamiltonian $h$ with the following properties

$$
\begin{aligned}
& \text { - } h \geq 0, \\
& \text { - } \operatorname{ker}(h)=S_{R},
\end{aligned}
$$

since by construction $\operatorname{tr}\left[\rho_{R} h\right]=0$. This Hamiltonian can then be extended to the whole lattice by assigning such a surrounding region $R_{v}$ and a corresponding $h_{v}$ to every vertex $v$ and defining

$$
H=\sum_{v} h_{v} \otimes \mathbb{1} \backslash R_{v} .
$$

In this way we have again $H \geq 0$ and $\langle\varphi|H| \varphi\rangle=$ $\sum_{v} \operatorname{tr}\left[\rho_{R_{v}} h_{v}\right]=0$. The Hamiltonian is thus local and frustration free [1, 2], in the sense that $|\varphi\rangle$ minimizes the energy locally for every interaction term $h_{v}$. The state is therefore sometimes called an optimum ground state [11]. We remark that it was recently proven in 14] that all local gapped Hamiltonians can be efficiently approximated by frustration free ones.

\section{INJECTIVITY}

We will now discuss the main condition which will later on turn out to be sufficient for proving uniqueness of the ground state for Hamiltonians of the form in Eq.(3). Consider a PEPS $|\varphi\rangle$ given by tensors $A_{i}$ (depending on the 
vertex). Let $R$ be a connected region on the graph containing $|R|$ sites (vertices) and having $e_{R}$ outgoing edges (bonds). The linear map $\Gamma_{R}: \mathbb{C}^{D^{e_{R}}} \rightarrow \mathbb{C}^{d^{|R|}}$ defined by

$$
\Gamma_{R}(C)=\sum_{i_{R}} \mathcal{C}\left[A_{i_{R}} C\right]\left|i_{R}\right\rangle
$$

maps the virtual boundary of $R$ to the physical bulk. Here $A_{i_{R}}$ stands for the tensor obtained by contracting all the $A_{i}$ 's in the region $R$ with bonds open in the boundary of $R$ (and then $C$ is a tensor with the same number $e_{R}$ of bonds). Graphically we will represent $\mathcal{C}\left[A_{i_{R}} C\right]$ as

$$
A_{i_{R}}-C
$$

where we have joined all the $e_{R}$ open bonds of $A_{i_{R}}$ and $C$ in one line.

If $\Gamma_{R}$ is an injective map, we will simply say that the region $R$ is injective. Similarly, if there is a proper covering of disjoint injective regions for the entire graph we will call the PEPS itself injective.

Of course, the injectivity property of $R$ is equivalent to the fact that the finite set $\left\{A_{i_{R}}\right\}_{i_{R}}$ generates the space of tensors with $e_{R} D$-dimensional indices. Just counting degrees of freedom one can see that the dimension of the space of tensors that we have to generate grows like the boundary $D^{e_{R}}$, whereas the number of tensors that we have grows like the volume $d^{|R|}$. This strongly suggest that the injectivity condition is generic on any lattice, i.e., almost always fulfilled as long as $d^{|R|} \geq D^{e_{R}}$. Indeed, if one takes a numerically random PEPS, it appears to be fulfilled in all the cases. As a more concrete example, one can also check that the 2D AKLT state [4] is injective.

In the remaining part of this section we will prove a pair of Lemmas which are particularly concerned with lifting the injectivity property and its consequences from smaller to larger regions. The reader not interested in the technical details may skip this part.

Lemma 1. If $R$ and $S$ are two disjoint injective regions, then $R \cup S$ is also injective.

Proof. We start from the picture

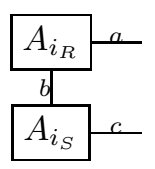

Since both $A_{i_{R}}$ and $A_{i_{S}}$ generate the respective space of tensors, then, summing in $i_{R}$ and $i_{S}$ we can get

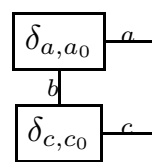

for each $a_{0}$ and $c_{0}$. Hence, we have injectivity in the region $R \cup S$, since we can generate all the canonical basis vectors of the respective space of tensors.
Let us call $G_{R}=\operatorname{range}\left(\Gamma_{R}\right)$ the subspace generated in the physical bulk by acting on the virtual boundary. Note that the reduced density operator $\rho_{R}$ is supported in $G_{R} \supseteq S_{R}$. Moreover, if $R$ and its complement are injective then the converse holds as well so that $G_{R}=S_{R}$.

Lemma 2. Let $R_{1}, R_{2}, R_{3}$ be three disjoint regions, and $\mathcal{H}_{R}=\mathbb{C}^{d^{|R|}}$ denote the total Hilbert space corresponding to region $R$.

1. $G_{R_{1} \cup R_{2} \cup R_{3}} \subseteq\left(G_{R_{1} \cup R_{2}} \otimes \mathcal{H}_{R_{3}}\right) \cap\left(\mathcal{H}_{R_{1}} \otimes G_{R_{2} \cup R_{3}}\right)$.

2. If $R_{1}$ and $R_{3}$ are not connected and $R_{2}$ and $R_{3}$ are both injective, then $G_{R_{1} \cup R_{2} \cup R_{3}}=$ $\left(G_{R_{1} \cup R_{2}} \otimes \mathcal{H}_{R_{3}}\right) \cap\left(\mathcal{H}_{R_{1}} \otimes G_{R_{2} \cup R_{3}}\right)$.

3. If all three regions are injective, then $G_{R_{1} \cup R_{2} \cup R_{3}}=\left(G_{R_{1} \cup R_{2}} \otimes \mathcal{H}_{R_{3}}\right) \cap\left(\mathcal{H}_{R_{1}} \otimes G_{R_{2} \cup R_{3}}\right) \cap$ $\left(G_{R_{1} \cup R_{3}} \otimes \mathcal{H}_{R_{2}}\right)$.

Proof. It is clear that $|\psi\rangle \in G_{R_{1} \cup R_{2}} \otimes \mathcal{H}_{R_{3}}$ if and only if $|\psi\rangle$ can be written as $\sum_{i_{R_{3}}} \Gamma_{R_{1} \cup R_{2}}\left(C_{i_{R_{3}}}\right)\left|i_{R_{3}}\right\rangle$. Then, if $|\psi\rangle \in G_{R_{1} \cup R_{2} \cup R_{3}}$, since we can write $|\psi\rangle=$ $\sum_{i_{R_{1}}, i_{R_{2}}, i_{R_{3}}} \mathcal{C}\left(A_{R_{1}} A_{R_{2}} A_{R_{3}} X\right)\left|i_{R_{1}} i_{R_{2}} i_{R_{3}}\right\rangle$, calling $C_{i_{R_{3}}}=$ $\mathcal{C}\left(A_{i_{R_{3}}} X\right)$, we have that $|\psi\rangle \in G_{R_{1} \cup R_{2}} \otimes \mathcal{H}_{R_{3}}$.

Of course, the same can be done for $\mathcal{H}_{R_{1}} \otimes G_{R_{2} \cup R_{3}}$, which proves part 1 . of the Lemma.

For the other parts note that if $|\psi\rangle \in$ $\left(G_{R_{1} \cup R_{2}} \otimes \mathcal{H}_{R_{3}}\right) \cap\left(\mathcal{H}_{R_{1}} \otimes G_{R_{2} \cup R_{3}}\right)$, then

$$
|\psi\rangle=\sum_{i_{R_{3}}} \Gamma_{R_{1} \cup R_{2}}\left(D_{i_{R_{3}}}\right)\left|i_{R_{3}}\right\rangle=\sum_{i_{R_{1}}} \Gamma_{R_{2} \cup R_{3}}\left(C_{i_{R_{1}}}\right)\left|i_{R_{1}}\right\rangle,
$$

which implies that

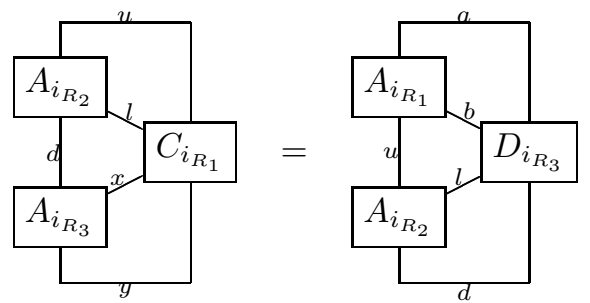

for every $i_{R_{1}}, i_{R_{2}}, i_{R_{3}}$. Or equivalently (summing in the repeated indices),

$$
A_{i_{R_{2}}}^{u, l, d} A_{i_{R_{3}}}^{d, x, y} C_{i_{R_{1}}}^{u, l, x, y}=A_{i_{R_{1}}}^{a, b, u} A_{i_{R_{2}}}^{u, l, d} D_{i_{R_{3}}}^{a, b, l, d},
$$

where the sum over $a$ and $y$ is only non-trivial if $R_{1}$ is not separated from $R_{3}$.

In order to prove 2 . we now use injectivity in $R_{2}$ and $R_{3}$. That is, for every fixed set of indices $l_{0}, u_{0}, d_{0}$ and $x_{0}, y_{0}$ there exist $\alpha_{i_{R_{2}}}^{u_{0}, l_{0}, d_{0}}$ and $\omega_{i_{R_{3}}}^{x_{0}, y_{0}}$ such that

$$
\begin{aligned}
\sum_{i_{R_{2}}} \alpha_{i_{R_{2}}}^{u_{0}, l_{0}, d_{0}} A_{i_{R_{2}}}^{u, l, d} & =\delta_{u, u_{0}} \delta_{l, l_{0}} \delta_{d, d_{0}}, \\
\sum_{i_{R_{3}}} \omega_{i_{R_{3}}}^{x_{0}, y_{0}} A_{i_{R_{3}}}^{d, x, y} & =\delta_{x, x_{0}} \delta_{y, y_{0}} .
\end{aligned}
$$


Inserting this into Eq.(15) we get that

$$
C_{i_{R_{1}}}^{u, l, x, y}=A_{i_{R_{1}}}^{a, b, u} \underbrace{\sum_{i_{R_{3}}} \omega_{i_{R_{3}}}^{x, y} D_{i_{R_{3}}}^{a, b, l, d}}_{M^{a, b, l, x, y}},
$$

(note that $M^{a, b, l, x, y}$ does not depend on $d$ ). Hence, the coefficients of $\psi$ have the form of the left part of the following identity (proven below)

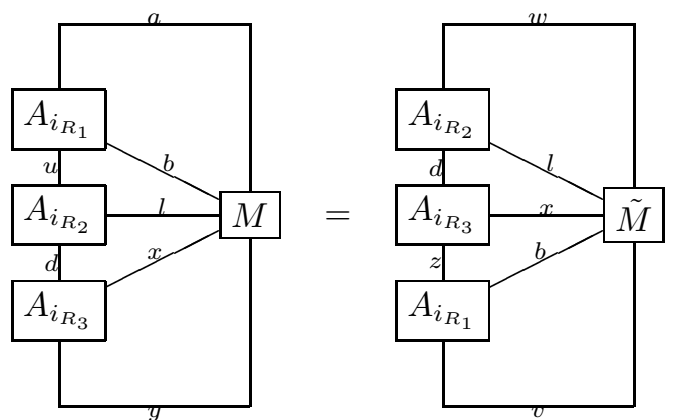

which implies that under the conditions of part $2 .|\psi\rangle \in$ $G_{R_{1} \cup R_{2} \cup R_{3}}$ completing the proof of the second part.

For 3 . we first repeat the above proof with a cyclic permutation of the regions, i.e., $\left(R_{1}, R_{2}, R_{3}\right) \rightarrow\left(R_{2}, R_{3}, R_{1}\right)$ leading to the identity in (8). Then we use injectivity in all three regions in order to replace $A_{i_{R_{1}}}^{a, b, u}$, $A_{i_{R_{2}}}^{u, l, d}$ and $A_{i_{R_{3}}}^{d, x, y}$ by $\delta_{a, a_{0}} \delta_{b, b_{0}}, \delta_{l, l_{0}}$ and $\delta_{x, x_{0}} \delta_{y, y_{0}}$ respectively. Inserting this into Eq. (8) we obtain $M^{a, b, l, x, y}=$ $\delta_{a, y} \tilde{M}^{w, l, x, b, v}$ so that

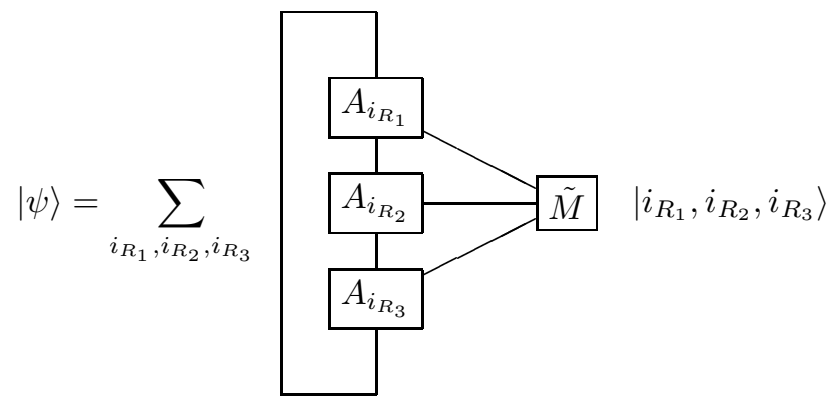

is indeed in $G_{R_{1} \cup R_{2} \cup R_{3}}$.

\section{UNIQUENESS OF THE GROUND STATE}

Our aim now is to prove that every injective $|\varphi\rangle$ is the unique ground state of a Hamiltonian of the form in Eq.(3). Consider an injective tile of the lattice, i.e., a covering by disjoint injective regions and let us merge sites within each injective region such that in the new, regrouped lattice each single vertex is injective. We connect the vertices in this super-lattice if the respective regions were connected before (see Fig.1). As a Hamiltonian we choose

$$
H=\sum_{(\alpha, \beta)} h_{\alpha, \beta} \otimes \mathbb{1}
$$

where the sum runs over all edges $(\alpha, \beta)$ in the superlattice and $h_{\alpha, \beta} \geq 0$ is any nearest-neighbor interaction term satisfying $\operatorname{ker}\left(h_{\alpha, \beta}\right)=G_{R_{\alpha} \cup R_{\beta}}$.

Theorem 3. For every injective PEPS on an arbitrary lattice there is a local frustration-free Hamiltonian such that the state is its unique ground state.

Remark: Note that though Eq.(9) gives a standard construction this Hamiltonian is not unique. We may in particular choose $h_{\alpha, \beta}^{\prime}=h_{\alpha, \beta}+h_{\alpha, \beta} Q h_{\alpha, \beta}$ for any $Q \geq 0$. Similarly, we can use the same construction for larger regions, or sometimes even for smaller ones for which injectivity does not yet hold (see below). The advantage of the latter is to reduce the number of sites involved in the interaction, as done for instance in the AKLT model.

Proof. Using the Hamiltonian of Eq.(9) and writing, with a slight abuse of notation, $G_{R}$ for $G_{R} \otimes \mathcal{H}_{\backslash R}$ we have to show that

$$
\bigcap_{(\alpha, \beta)} G_{R_{\alpha} \cup R_{\beta}}=G_{\cup_{\alpha} R_{\alpha}}
$$

as the left hand side is the ground state space of $H$ and the right hand side is the one-dimensional space corresponding to the PEPS. We will show this identity by induction. To this end consider a collection of disjoint regions $\cup_{i=0}^{N} A_{i}=A$ such that $A_{1}, \ldots, A_{N}$ are connected to a region $B$ (all regions being injective). Then as $G_{A} \subseteq G_{A_{0} \cup A_{i}}$ we have that

$$
G_{A} \bigcap_{i=1}^{N} G_{A_{i} \cup B} \subseteq \bigcap_{i=1}^{N}\left(G_{A_{0} \cup A_{i}} \cap G_{A_{i} \cup B}\right)=\bigcap_{i=1}^{N} G_{A_{0} \cup A_{i} \cup B},
$$

where the last equality follows from part 2. of Lemma 2. If we exploit in addition that $G_{A} \subseteq G_{\cup_{i \in I} A_{i}}$ for any $I \subseteq\{0, \ldots, N\}$ together with part 3 . of Lemma 2 we obtain

$$
G_{A} \bigcap_{i=1}^{N} G_{A_{i} \cup B} \subseteq G_{A \cup B}
$$

Eq. (12) can now be used as an induction step in order to show that the l.h.s. of Eq. (10) is contained in the r.h.s. . The converse inclusion follows, however, from part 1 . of Lemma 2 which completes the proof.

\section{QUANTUM STATES FROM CLASSICAL MODELS}

In [8] we constructed PEPS from any classical spin model with nearest-neighbor interaction (with $d$ possible configurations per site and a fixed given temperature $\frac{1}{\beta}$ ) in such a way that (i) the physical dimension coincides with the bond dimension, i.e., $d=D$; and (ii) the PEPS reproduces the correlations of the classical thermal state. 
In a sense, this map replaces thermal by quantum fluctuations. In this section we will analyze in which cases PEPS constructed in this way are unique ground states of their parent Hamiltonians from Eq.(9).

First of all we will review the construction of [8] For a classical Hamiltonian of the form $H(x)=$ $\sum_{(i, j)} h\left(x_{i}, x_{j}\right)$, with $x_{i}=1, \ldots, d$ we define the associate PEPS as

$$
|\psi\rangle=\frac{1}{\sqrt{Z}} \exp \left[-\frac{\beta}{2} \sum_{(i, j)} \hat{h}_{i j}\right]|+\cdots+\rangle,
$$

where $|+\rangle=\sum_{x=1}^{d}|x\rangle$ and $\hat{h}_{i j}$ is a diagonal operator that acts as $\hat{h}_{i j}\left|x_{i} x_{j}\right\rangle=h\left(x_{i}, x_{j}\right)\left|x_{i} x_{j}\right\rangle$. To see explicitly the PEPS structure of $|\psi\rangle$ one can notice that the nonunitary gate $\exp \left[-\frac{\beta}{2} \hat{h}_{i j}\right]$ is indeed equal to $P_{i} \otimes P_{j}|I\rangle$, where $|I\rangle=\sum_{x=1}^{d}|x x\rangle$ is an auxiliary maximally entangled state between the sites $i$ and $j$ and $P_{i}: \mathcal{C}^{d^{2}} \longrightarrow \mathcal{C}^{d}$ is an operator (from the joint physical-virtual system into the physical system) acting as $P_{i}\left|x_{i} v\right\rangle=\left|x_{i}\right\rangle\left\langle\phi_{x_{i}}^{i} \mid v\right\rangle$. Here the vectors $\left|\phi_{x_{i}}^{i}\right\rangle$ can be obtained by a SVD to verify $\exp \left[-\frac{\beta}{2} h\left(x_{i}, x_{j}\right)\right]=\sum_{k}\left\langle\phi_{x_{i}}^{i} \mid k\right\rangle\left\langle k \mid \phi_{x_{j}}^{j}\right\rangle$. Then, the tensors defining the PEPS $|\psi\rangle$ are

$$
A_{x_{i} ; \alpha_{1} \ldots \alpha_{n}}^{i}=\prod_{e}\left\langle\phi_{x_{i}}^{e} \mid \alpha_{e}\right\rangle, \quad \alpha_{e}=1, \ldots, d,
$$

where $\left|\phi_{x_{i}}^{e}\right\rangle$ may, of course, depend on the lattice site $i$, and the product is taken over all edges $e$ connected with this site. Generically, $\phi^{e}$ can be considered (via $\left.\left\langle x_{i}\right| \mapsto\left\langle\phi_{x_{i}}^{e}\right|\right)$ an invertible $d \times d$ matrix. For instance, for the Ising model $H(x)=-\sum_{(i, j)} x_{i} x_{j}, x_{i}= \pm 1$,

$$
\phi^{e}=\phi=\left(\begin{array}{cc}
\sqrt{\sinh \frac{\beta}{2}} & \sqrt{\cosh \frac{\beta}{2}} \\
-\sqrt{\sinh \frac{\beta}{2}} & \sqrt{\cosh \frac{\beta}{2}}
\end{array}\right),
$$

which is indeed invertible for all $\beta$.

\section{A. Injectivity and Criticality}

Surprisingly injectivity does, in the case of PEPS corresponding to classical models, not depend on the type of interaction, but merely on the lattice geometry of the PEPS, which coincides with the interaction graph of the classical model. If a subset on the interaction graph is such that every site has at most one outgoing edge, then the corresponding region has the injectivity propertyotherwise it does not. The simple reason is as follows.

Consider a region $R$ on the interaction graph and let $E$ be the set of edges connecting sites on the boundary $\bar{R} \subseteq$ $R$ to exterior sites. We are interested in the injectivity of the operator $\Gamma_{R}: \mathbb{C}^{D \otimes|E|} \rightarrow \mathbb{C}^{d \otimes|R|}$. The product form of the $A$ 's (13) leads to

$$
\left\langle x\left|\Gamma_{R}\right| \bar{\alpha}\right\rangle=C(x)\langle\bar{x}|F| \bar{\alpha}\rangle,
$$

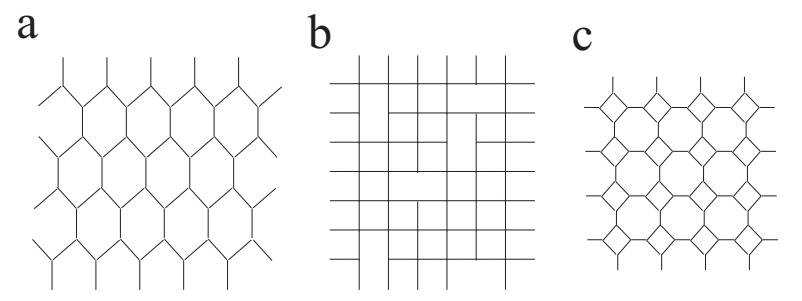

FIG. 2: Examples of 2D lattice geometries leading to injectivity when the PEPS is constructed from a classical spin model with corresponding interaction graph. A 3D example would be a tetrahedral crystal lattice (ice).

where the bar always means "at the boundary" and $C(x)$ is the result of the contractions along all $e \notin E$. In the case $|E|=|\bar{R}|$ we have that the vectors $\bar{x}$ and $\bar{\alpha}$ have the same dimension and $F=\otimes_{e \in E} \phi^{e}$.

Hence, $F$ is invertible so that $\Gamma_{R}$ is injective [19]. On the other hand if $|E|>|\bar{R}|$ then $F$ is rectangular and not invertible, such that $\Gamma_{R}$ cannot be injective. Hence, we do not have injectivity for the square lattice but we do have it e.g. for the tetrahedral 3D or the hexagonal 2D lattice (Figure 22a). A 2D square lattice can as well lead to injectivity if we either introduce a substructure at each point (Figure 2 $\mathrm{c}$ ) or if we allow for defects (Figure 2.b): if with probability $p$ a bond is missing then injective regions contain of the order of $(1-p)^{2} / p^{3}$ lattice sites.

With this at hand we can now give an example of an injective PEPS whose associated parent Hamiltonian is gapless. It is the PEPS associated to the 2D classical (isotropic) hexagonal Ising model. On the one hand, we have injectivity and hence the PEPS is the unique ground state of its parent Hamiltonian. On the other hand, the Ising model becomes critical for $\beta=\frac{1}{2} \ln (2+\sqrt{3})$ [15], which implies that the PEPS has correlations with powerlaw decay. By [16], this implies that the parent Hamiltonian has to be gapless.

\section{B. Uniqueness for non-injective lattices}

Although injectivity is a generic and powerful condition for proving uniqueness of the ground state it is not a necessary requirement. In fact, in the case of a PEPS associated to a classical model on a square lattice, although we do not have injectivity, it is still the unique ground state of its parent Hamiltonian [20]. The reason is that we can essentially follow the main proof given above without using injectivity. The only thing we need for that is to show that the intersection properties of the ranges $G_{R}$ in Lemma 2 remain true. This can be shown easily just using (14) and some dimension considerations. We will illustrate them in a particular case, that is, we 
will prove that, with the obvious notation,

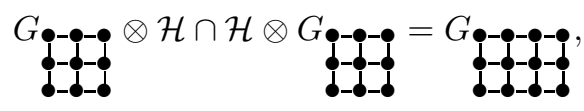

with each dot corresponding to a two-dimensional Hilbert space.

Since the inclusion $\supset$ is trivial it is enough to show that the dimension of the left hand side is $\leq 2^{10}$ (that, by (14) is the dimension of the right hand side). Now, the operator $\mathbb{1} \otimes|0\rangle_{a}\left\langle\left. 0\right|_{a}\right.$ acting on

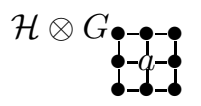

has trivial kernel, where $a$ is the position $(2,3)$. To see this, it is enough to notice that by (14),

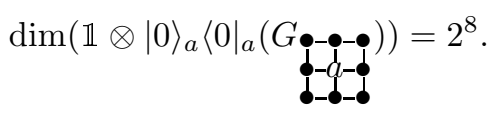

In particular $\mathbb{1} \otimes|0\rangle_{a}\left\langle\left. 0\right|_{a}\right.$ has also trivial kernel when acting on the left hand side of (15). Therefore, the dimension of this left hand side is

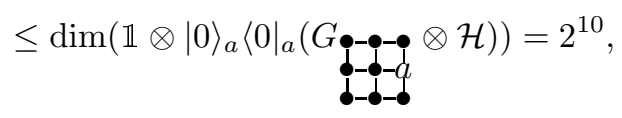

where the last equality comes from the fact that, again by (14),

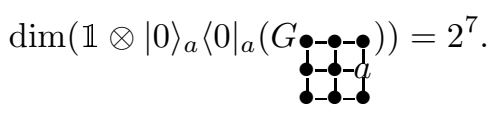

Finally, it has to be noticed that, if we consider the following regions,

$$
R_{1}=\because \because \bullet \quad R_{2}=\bullet \bullet \bullet,
$$

by dimensional considerations using (14) as above, it is straight forward to verify that $P_{R_{1}}=P_{R_{2}} \otimes \mathbb{1}$, where $P_{R}$ is the projection onto the orthogonal complement of $G_{R}$. Then, the parent Hamiltonian of our PEPS can be considered to be both $P_{R_{1}}$ or $P_{R_{2}}$. While $P_{R_{1}}$ has still a square structure, $P_{R_{2}}$ is more natural in the sense that it reflects exactly the nearest neighbors of the spin in the center of a cross (for this reason it is the one that will appear in the next section).

\section{A COMPUTABLE SUFFICIENT CONDITION FOR AN ENERGY GAP}

The detection of either criticality or the occurrence of a spectral gap is an important problem in both condensed matter theory and quantum information theory. Unfortunately in the 2D situation there are very few criteria for the existence of a gap above the ground state energy. In this section we will provide a computable sufficient condition for a gap in the parent Hamiltonian of a PEPS. The idea comes from the 1D case [2] and the proof is essentially the same. For simplicity we consider a translational invariant frustration free local Hamiltonian on a $2 \mathrm{D}$ square lattice. Let $h$ be a locally acting projector and $h_{i j}$ its translate by $i$ columns and $j$ rows so that $H=\sum_{i j} h_{i j}$. Since $H$ is local, there exists a small $I \subset\{1, \ldots, N\} \times\{1, \ldots, M\}$ such that $h h_{i j} \geq 0$ whenever $(i, j) \notin I$. Then,

Proposition 4. If $H$ has a unique ground state (i.e., it is the parent Hamiltonian of an injective PEPS) and

$$
\sum_{(i, j) \in I} h h_{i j}+h_{i j} h>-\frac{1}{|I|+1}\left(h+\sum_{(i, j) \in I} h_{i j}\right)
$$

then there is an $\epsilon>0$ such that $H^{2}>\epsilon H$ and hence there is a uniform (independent of the size of the system) energy gap for $H$.

In general, one can replace the right hand side of (16) by

$$
-\frac{1}{\sum \alpha_{i j}}\left(\alpha_{00} h+\sum_{(i, j) \in I} \alpha_{i j} h_{i j}\right) .
$$

In the case of the PEPS $|\psi\rangle$ associated to the classical Ising model on the square lattice, the above criterion finds a gap for $\beta<0.27$. In this case one can indeed show analytically the existence of an energy gap for the whole range $\beta<\beta_{c}=\frac{1}{2}(1+\sqrt{2})$ [8].

To do so we need to use the concept of a Q-matrix: a matrix such that $q_{i i} \leq 0$ for all $i, q_{i j} \geq 0$ for all $i \neq j$, and $\sum_{i} q_{i j}=0$ for all $j$. As a consequence of [17, Thm 2.1.2] the eigenvalues of $Q$ verify $0=\lambda_{0} \geq \lambda_{1} \geq \ldots \geq$ $\lambda_{n-1} \geq-1$. The Q-matrix we are going to use is the one that generates the Glauber dynamics associated to the classical Ising model [18]. That is, for a function $f$ on the configuration space we define the gradient of a function $f$ at the site $i$ as the function $\nabla_{i} f(x)=f\left(x^{i}\right)-f(x)$, where $x^{i}$ is the configuration $x$ after flipping the spin at site $i$. With this notation we define

$$
Q f(x)=\sum_{i} c(i, x) \nabla_{i} f(x)
$$

where the sum is on the sites $i$ of the lattice and the transition rates $c(i, x)$ are those associated to the Metropolis dynamics

$$
c(i, x)=\min \left\{1, e^{-\beta\left(\nabla_{i} H_{i}\right)(x)}\right\},
$$

with $H_{i}(x)=-\sum_{j} x_{i} x_{j}$ (the sum on the $j$ 's connected by $i$ in the interaction graph; in the case of the square lattice the nearest neighbors of $i$ ).

$Q$ is trivially a $Q$-matrix, and it is proven in [18] that $\operatorname{gap}(Q)=-\lambda_{1}$ has a uniform (independent of the size of 
the system) lower bound. Now we consider the Hamiltonian in our quantum system given by $H_{Q}=-Q$. If we define the matrices $Q_{i}$ by

$$
\left\langle y\left|Q_{i}\right| x\right\rangle=\left\{\begin{array}{c}
-c(i, x), \quad y=x \\
c(i, x), \quad y=x^{i} \\
0, \quad \text { else }
\end{array}\right.
$$

we have that

(i) $Q_{i}$ is a $Q$-matrix (and hence $\leq 0$ ),

(ii) $Q=\sum_{i} Q_{i}$,

(iii) $Q_{i}$ acts only on the nearest neighbors of $i$,

(iv) $Q|\psi\rangle=0$ [17, Thm. 3.5.5] and hence $Q_{i}|\psi\rangle=0$ for all $i$.

With these properties and using (13) it is easy to show that $H_{Q}$ is in matrix ordering upper bounded by the parent Hamiltonian of our PEPS. This immediately implies that the parent Hamiltonian is gapped.

Acknowledgements: D.P.-G. was supported by the Spanish grants MTM2005-00082 and Ramon y Cajal.

\section{APPENDIX: SITE-INDEPENDENT TENSORS}

Here we prove that for every PEPS which is translational invariant on a $N \times M 2 \mathrm{D}$ square lattice there exists a PEPS representation with site-independent tensors. The proof follows closely the 1D case [1] .
For doing tensor contraction we can apply Dirac notation along the edges of the lattice, but we will need some conventions. The first is that, when we do a horizontal (resp. vertical) contraction, the vertical (resp. horizontal) indices always tensorize. For example, the result from contracting the positions $(1,1)$ and $(1,2)$ will be

$$
\sum_{\substack{l_{1}, u_{1}, d_{1} \\ r_{2}, u_{2}, d_{2} \\ a}} A_{l_{1}, a, u_{1}, d_{1}}^{(1,1)} A_{a, r_{2}, u_{2}, d_{2}}^{(1,2)}\left|l_{1}\right\rangle_{h}\left\langle\left. r_{2}\right|_{h} \mid u_{1}, u_{2}\right\rangle_{v}\left\langle d_{1},\left.d_{2}\right|_{v} .\right.
$$

The other convention is that the edges of each row (column) are also joined.

We start with a general PEPS:

$$
|\varphi\rangle=\sum_{i_{(1,1)}, \ldots, i_{(N, M)}} \mathcal{C}\left(\left(A_{i_{(j, k)}}^{(j, k)}\right)_{(j, k)}\right)\left|i_{(1,1)}\right\rangle \cdots\left|i_{(N, M)}\right\rangle
$$

and define site-independent tensors $(N M)^{\frac{1}{N M}} S_{i}$ as

$$
\sum_{u, d, l, r, j, k} A_{i ; u, d, l, r}^{(j, k)}|j, k, l\rangle_{h}\left\langle j, k+1,\left.r\right|_{h} \mid k, j, u\right\rangle_{v}\left\langle k, j+1,\left.d\right|_{v} .\right.
$$

With this choice translational invariance immediately implies that

$$
|\varphi\rangle=\sum_{i_{(1,1)}, \ldots, i_{(N, M)}} \mathcal{C}\left(\left(S_{i_{(j, k)}}\right)_{(j, k)}\right)\left|i_{(1,1)}\right\rangle \cdots\left|i_{(N, M)}\right\rangle .
$$

[1] D. Pérez-García, F. Verstraete, M.M. Wolf, J.I. Cirac, Quant. Inf. Comp. 7, 401 (2007); arxiv:quant-ph/0608197

[2] M. Fannes, B. Nachtergaele and R. F. Werner, Commun. Math. Phys. 144, 443-490 (1992).

[3] A. Klümper, A. Schadschneider, J. Zittartz, J. Phys. A 24, L955 (1991); Z. Phys. B 87, 281 (1992).

[4] I. Affleck, T. Kennedy, E. H. Lieb and H. Tasaki, Commun. Math. Phys. 115, 477 (1988).

[5] F. Verstraete, J.I. Cirac, J.I. Latorre, E. Rico and M.M. Wolf, Phys. Rev. Lett. 94, 140601 (2005); C. Schoen, E. Solano, F. Verstraete, J. I. Cirac, M. M. Wolf, Phys. Rev. Lett. 95, 110503 (2005); M.M. Wolf, G. Ortiz, F. Verstraete, J.I. Cirac, Phys. Rev. Lett. 97, 110403 (2006); R. Josza, arXiv:quant-ph/0603163 G. Vidal, Phys. Rev. Lett. 91, 147902 (2003); F. Verstraete and I. Cirac, Phys. Rev. B 73, 094423 (2006); D. Gross, J. Eisert, N. Schuch, D. Perez-Garcia, arXiv:0706.3401 (2007); M.B. Hastings, arXiv:cond-mat/0701055 (2007).

[6] F. Verstraete, D. Porras and J.I. Cirac, Phys. Rev. Lett. 93, 227205 (2004); M. Zwolak, G. Vidal, Phys. Rev. Lett. 93, 207205 (2004); T.J. Osborne, Phys. Rev. Lett. 97157202 (2006); G. Vidal, arXiv:cond-mat/0512165 (2005); F. Verstraete, A. Weichselbaum, U. Schollwöck, J.I. Cirac, J. von Delft, arXiv:cond-mat/0504305 (2005).
[7] F. Verstraete, J.I. Cirac, cond-mat/0407066 (2004).

[8] F. Verstraete, M.M. Wolf, D. Pérez-García, J.I. Cirac, Phys. Rev. Lett. 96, 220601 (2006).

[9] N. Schuch, M. M. Wolf, F. Verstraete, J. I. Cirac, Phys. Rev. Lett. 98, 140506 (2007).

[10] B.S. Shastry, B. Sutherland, Physica B 108, 1069 (1981); H.J. Schmidt, J. Phys. A 38, 2123 (2005); B. Kumar, Phys. Rev. B 66, 024406 (2002).

[11] H. Niggemann, A. Klümper, J. Zittartz, Eur. Phys. J. B 13, 15 (2000); Z. Phys. B 104, 103 (1997).

[12] F. Verstraete, J.I. Cirac, Phys. Rev. A 70, 060302(R) (2004).

[13] V. Murg, F. Verstraete, J.I. Cirac, Phys. Rev. A 75, 033605 (2007).

[14] M.B. Hastings, Phys. Rev. B 73, 085115 (2006)

[15] V. Privman, M. Fisher, Phys. Rev. B 30, 322 (1984); K. Husimi, I. Syozi, Prog. Theor. Phys. 5, 177 (1950).

[16] M. Hastings, T. Koma, Commun.Math.Phys. 265781 (2006); B. Nachtergaele, R. Sims Commun. Math. Phys., 265119 (2006).

[17] J. R. Norris, Markov Chains, Cambridge University Press, Cambridge 1997.

[18] F. Martinelli, Lectures on Glauber Dynamics for Discrete Spin Models, LNM 1717, Springer, 1999; F. Martinelli, E. Olivieri, Comm. Math. Phys. 161, 447 (1994); 
F. Martinelli, E. Olivieri, R. Schonmann, Comm. Math. Phys. 165, 33 (1994); F. Martinelli, A. Sinclair, D. Weitz, Comm. Math. Phys. 250, 301 (2004).

[19] Strictly speaking this is only true if for each $\bar{x} \in \bar{R}$ there is at least one configuration $x$ such that $C(x) \neq 0$. This should again be true in the "generic" case. For the Ising model one can indeed show analytically that $C(x) \neq 0$ for all configurations $x$ and all values of $\beta$ (including the critical point).

[20] We are assuming again the generic conditions that the matrices $\phi^{e}$ are invertible and that $C(x) \neq 0$ for all configurations $x$, which is true e.g. for the Ising model. 